\title{
A Closed-Form Solution of Spatial Sliders for Rigid-Body Guidance
}

\author{
C. Huang, W. C. Huang and G. Kiper
}

\begin{abstract}
This paper presents a closed-form solution to the four-position synthesis problem by using a spatial slider, which is a spatial dyad of two perpendicularly intersected cylindrical joints. We utilize the dialytic elimination method to simplify the synthesis equations and to obtain a univariate ninth degree polynomial equation. Among the nine sets of solutions, two of them are infinite, and one is the displacement screw from the first position to the second position. Therefore, we have at most six real solutions that can be used to design spatial sliders for the four-position synthesis problem. A numerical example is provided in to demonstrate the validity of the solution procedure.
\end{abstract}

Key words: Spatial slider, cylindrical joint, rigid-body guidance, dialytic elimination.

\section{Introduction}

The rigid-body guidance problem is the central problem in kinematic synthesis of linkages. In planar kinematics, given several positions of a body, one can design a crank or a slider to guide the body through the prescribed positions [1,2]. Spatial counterpart of the planar synthesis problem has been extensively studied too; various spatial dyads have been investigated for rigid-body guidance $[3,4,8]$. This paper

Chintien Huang

Department of Mechanical Engineering, National Cheng Kung University, Tainan, Taiwan, e-mail: chuang@mail.ncku.edu.tw

Weiche Huang

Department of Mechanical Engineering, National Cheng Kung University, Tainan, Taiwan, e-mail: tony77113@gmail.com

Gökhan Kiper

Department of Mechanical Engineering, İzmir Institute of Technology, İzmir, Turkey, e-mail: gokhankiper@iyte.edu.tr 
focuses on the spatial generalization of the planar slider, and we investigate the solution to the synthesis equations of the special cylindrical-cylindrical dyad, with the joint axes perpendicularly intersected, for rigid body guidance.

By using screw geometry, the spatial generalization of the synthesis of the planar crank has led to elegant geometric and algebraic results in computational kinematics $[3,5,6]$. Built upon these results, this paper utilizes a special screw triangle [4] to derive synthesis equations of the spatial slider and seeks to find all possible solutions to the design equations. However, due to the complexity of the equations, analytical solutions cannot be obtained. Instead, we utilize the dialytic elimination method [7] to find a closed-form solution, in which a univariate polynomial equation is obtained. The degree of the polynomial equation is the number of all possible solutions; however, we may need to exclude some extraneous roots.

This paper is organized as follows: first, we derive the synthesis equations and show that the maximum number of design positions is four. Second, we divide the equations into two uncoupled groups and use one of them to solve for the direction vectors of the joints. To further simplify the solution procedure, we superimpose two three-position problems instead of solving a four-position problem. Third, based on the obtained solutions of the direction vectors, we utilize the second group of equations to obtain the position vectors of points on the joint axes. Finally, a numerical example is provided to verify the solution procedure.

\section{Design Equations for the Synthesis of Spatial Sliders}

Figure 1 shows a spatial slider guiding a rigid body from one position to another. The spatial slider consists of two perpendicularly intersected cylindrical joints. The ground (fixed) and moving joints are denoted by $F$ and $M_{1}$, respectively. Let the displacement screw for displacing the body from position $\Sigma_{1}$ to position $\Sigma_{2}$ be denoted by $\$_{12}$, and the rotation and translation parameters be $\theta_{12}$ and $d_{12}$, respectively. The direction vector of $\$_{12}$ is denoted by $\hat{\mathbf{s}}_{12}$, and the position vector of a point on $\$_{12}$ is denoted by $\mathbf{A}_{12}$. The geometry of the relation among $F, M_{1}$, and $\$_{12}$ is a special screw triangle illustrated in Figure 2. The following equations can be obtained based on the geometric relation of the screw triangle [4]:

$$
\begin{gathered}
\hat{\mathbf{F}} \cdot \hat{\mathbf{M}}_{1}=0 \\
\hat{\mathbf{F}} \cdot\left(\hat{\mathbf{s}}_{12} \times \hat{\mathbf{M}}_{1}\right)+\tan \frac{\theta_{12}}{2}\left(\hat{\mathbf{F}} \times \hat{\mathbf{s}}_{12}\right) \cdot\left(\hat{\mathbf{s}}_{12} \times \hat{\mathbf{M}}_{1}\right)=0 \\
\hat{\mathbf{F}} \cdot\left(\mathbf{Q}_{1} \times \hat{\mathbf{M}}_{1}\right)+\hat{\mathbf{M}}_{1} \cdot(\mathbf{G} \times \hat{\mathbf{F}})=0 \\
{\left[1-\left(\hat{\mathbf{s}}_{12} \cdot \hat{\mathbf{F}}\right)^{2}\right] \cdot\left\{\left[\hat{\mathbf{s}}_{12}-\left(\hat{\mathbf{s}}_{12} \cdot \hat{\mathbf{M}}_{1}\right) \hat{\mathbf{M}}_{1}\right] \cdot\left(\mathbf{Q}_{1}-\mathbf{A}_{12}\right)\right\}-\left[1-\left(\hat{\mathbf{s}}_{12} \cdot \hat{\mathbf{M}}_{1}\right)^{2}\right] \cdot\left\{\left[\hat{\mathbf{s}}_{12}\right.\right.} \\
\left.\left.-\left(\hat{\mathbf{s}}_{12} \cdot \hat{\mathbf{F}}\right) \hat{\mathbf{F}}\right] \cdot\left(\mathbf{G}-\mathbf{A}_{12}\right)\right\}+\frac{d_{12}}{2}\left[1-\left(\hat{\mathbf{s}}_{12} \cdot \hat{\mathbf{F}}\right)^{2}\right] \cdot\left[1-\left(\hat{\mathbf{s}}_{12} \cdot \hat{\mathbf{M}}_{1}\right)^{2}\right]=0 \\
\hat{\mathbf{M}}_{1}^{2}=m_{1 x}^{2}+m_{1 y}^{2}+m_{1 z}^{2}=1
\end{gathered}
$$




$$
\begin{gathered}
\hat{\mathbf{F}}^{2}=f_{x}^{2}+f_{y}^{2}+f_{z}^{2}=1 \\
\hat{\mathbf{M}}_{1} \cdot \mathbf{Q}_{1}=m_{1 x} \cdot q_{1 x}+m_{1 y} \cdot q_{1 y}+m_{1 z} \cdot q_{1 z}=0 \\
\hat{\mathbf{F}} \cdot \mathbf{G}=f_{x} \cdot g_{x}+f_{y} \cdot g_{y}+f_{z} \cdot g_{z}=0
\end{gathered}
$$

Note that we use the symbol ^ to denote unit vectors or unit screws. The unknowns in the above equations are the direction vector of the moving joint $\hat{\mathbf{M}}_{1}\left(m_{1 x}, m_{1 y}, m_{1 z}\right)$, the position vector of a point on the moving axis $\mathbf{Q}_{1}\left(q_{1 x}, q_{1 y}, q_{1 z}\right)$, the direction vector of the fixed joint $\hat{\mathbf{F}}\left(f_{x}, f_{y}, f_{z}\right)$, and the position vector of a point on the fixed axis $\mathbf{G}\left(g_{x}, g_{y}, g_{z}\right)$. Eqs. (1) and (3) indicate that the joint axes are intersected perpendicularly. Eqs. (7) and (8) constrain the points on the axes in such a way that the position vectors of the points must be perpendicular to the joint direction vectors.

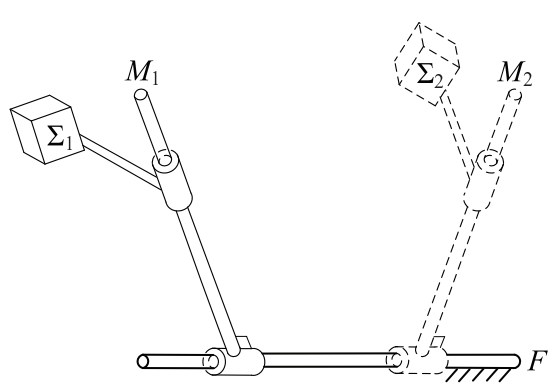

Fig. 1 A rigid body guided by a spatial slider

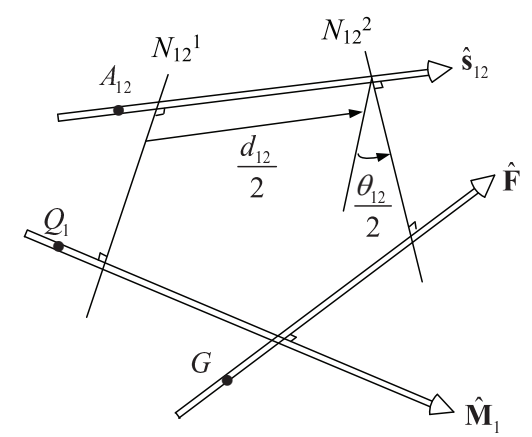

Fig. 2 Screw geometry of the spatial slider

There are 12 unknowns but only eight equations; therefore, we can specify two more positions by displacement screws $\$_{13}$ (with rotation $\theta_{13}$ and translation $d_{13}$ ) and $\$_{14}$ (with rotation $\theta_{14}$ and translation $d_{14}$ ). As a result, we have four more design equations as follows:

$$
\begin{gathered}
\hat{\mathbf{F}} \cdot\left(\hat{\mathbf{s}}_{13} \times \hat{\mathbf{M}}_{1}\right)+\tan \frac{\theta_{13}}{2}\left(\hat{\mathbf{F}} \times \hat{\mathbf{s}}_{13}\right) \cdot\left(\hat{\mathbf{s}}_{13} \times \hat{\mathbf{M}}_{1}\right)=0 \\
\hat{\mathbf{F}} \cdot\left(\hat{\mathbf{s}}_{14} \times \hat{\mathbf{M}}_{1}\right)+\tan \frac{\theta_{14}}{2}\left(\hat{\mathbf{F}} \times \hat{\mathbf{s}}_{14}\right) \cdot\left(\hat{\mathbf{s}}_{14} \times \hat{\mathbf{M}}_{1}\right)=0 \\
{\left[1-\left(\hat{\mathbf{s}}_{13} \cdot \hat{\mathbf{F}}\right)^{2}\right] \cdot\left\{\left[\hat{\mathbf{s}}_{13}-\left(\hat{\mathbf{s}}_{13} \cdot \hat{\mathbf{M}}_{1}\right) \hat{\mathbf{M}}_{1}\right] \cdot\left(\mathbf{Q}_{1}-\mathbf{A}_{13}\right)\right\}-\left[1-\left(\hat{\mathbf{s}}_{13} \cdot \hat{\mathbf{M}}_{1}\right)^{2}\right] \cdot\left\{\left[\hat{\mathbf{s}}_{13}\right.\right.} \\
\left.\left.-\left(\hat{\mathbf{s}}_{13} \cdot \hat{\mathbf{F}}\right) \hat{\mathbf{F}}\right] \cdot\left(\mathbf{G}-\mathbf{A}_{13}\right)\right\}+\frac{d_{13}}{2}\left[1-\left(\hat{\mathbf{s}}_{13} \cdot \hat{\mathbf{F}}\right)^{2}\right] \cdot\left[1-\left(\hat{\mathbf{s}}_{13} \cdot \hat{\mathbf{M}}_{1}\right)^{2}\right]=0 \\
{\left[1-\left(\hat{\mathbf{s}}_{14} \cdot \hat{\mathbf{F}}\right)^{2}\right] \cdot\left\{\left[\hat{\mathbf{s}}_{14}-\left(\hat{\mathbf{s}}_{14} \cdot \hat{\mathbf{M}}_{1}\right) \hat{\mathbf{M}}_{1}\right] \cdot\left(\mathbf{Q}_{1}-\mathbf{A}_{14}\right)\right\}-\left[1-\left(\hat{\mathbf{s}}_{14} \cdot \hat{\mathbf{M}}_{1}\right)^{2}\right] \cdot\left\{\left[\hat{\mathbf{s}}_{14}\right.\right.} \\
\left.\left.-\left(\hat{\mathbf{s}}_{14} \cdot \hat{\mathbf{F}}\right) \hat{\mathbf{F}}\right] \cdot\left(\mathbf{G}-\mathbf{A}_{14}\right)\right\}+\frac{d_{14}}{2}\left[1-\left(\hat{\mathbf{s}}_{14} \cdot \hat{\mathbf{F}}\right)^{2}\right] \cdot\left[1-\left(\hat{\mathbf{s}}_{14} \cdot \hat{\mathbf{M}}_{1}\right)^{2}\right]=0
\end{gathered}
$$




\section{Solutions of Direction Vectors of the Joint Axes}

The 12 design equations are too complicated to be solved simultaneously. However, we can deal with only three positions at a time and superimpose two threeposition problems. For example, we can first design the spatial slider to guide the body through positions 1,2 , and 3 and then design the spatial slider to guide the body through positions 1,2 , and 4 . Notice that the 12 equations can be decoupled by first solving Eqs. (1), (2), (5), (6), (9), and (10) for the direction vectors. We can then utilize the remaining equations to solve for the position vectors.

For positions 1, 2, and 3, substituting Eq. (1) into Eqs. (2) and (9) and rearranging the equations gives:

$$
\begin{aligned}
& {\left[\hat{\mathbf{s}}_{12} \times \hat{\mathbf{M}}_{1}+\tan \frac{\theta_{12}}{2}\left(\hat{\mathbf{s}}_{12} \cdot \hat{\mathbf{M}}_{1}\right) \hat{\mathbf{s}}_{12}\right] \cdot \hat{\mathbf{F}}=0} \\
& {\left[\hat{\mathbf{s}}_{13} \times \hat{\mathbf{M}}_{1}+\tan \frac{\theta_{13}}{2}\left(\hat{\mathbf{s}}_{13} \cdot \hat{\mathbf{M}}_{1}\right) \hat{\mathbf{s}}_{13}\right] \cdot \hat{\mathbf{F}}=0}
\end{aligned}
$$

We can rearrange Eqs. (1), (13), and (14) in a matrix form as follows:

$$
\left[\mathbf{K}_{m}\right]\left[\begin{array}{c}
f_{x} \\
f_{y} \\
f_{z}
\end{array}\right]=\left[\begin{array}{l}
0 \\
0 \\
0
\end{array}\right]
$$

Note that the unit direction vectors of the specified screws $\hat{\mathbf{s}}_{12}\left(s_{12 x}, s_{12 y}, s_{12 z}\right)$ and $\hat{\mathbf{s}}_{13}\left(s_{13 x}, s_{13 y}, s_{13 z}\right)$ are known parameters. For the linear system, Eq. (15), to have non-trivial solutions, the determinant of $\mathbf{K}_{m}$ must be zero:

$$
\begin{aligned}
\operatorname{det}\left(\mathbf{K}_{m}\right)_{123}= & \hat{\mathbf{M}}_{1} \cdot\left\{\left[\hat{\mathbf{s}}_{12} \times \hat{\mathbf{M}}_{1}+\tan \frac{\theta_{12}}{2}\left(\hat{\mathbf{s}}_{12} \cdot \hat{\mathbf{M}}_{1}\right) \hat{\mathbf{s}}_{12}\right]\right. \\
& \left.\times\left[\hat{\mathbf{s}}_{13} \times \hat{\mathbf{M}}_{1}+\tan \frac{\theta_{13}}{2}\left(\hat{\mathbf{s}}_{13} \cdot \hat{\mathbf{M}}_{1}\right) \hat{\mathbf{s}}_{13}\right]\right\} \\
= & 0
\end{aligned}
$$

Expanding the determinant gives:

$$
\begin{aligned}
a_{1} m_{1 x} m_{1 y}^{2} & +a_{2} m_{1 x}^{2} m_{1 y}+a_{3} m_{1 x}^{3}+a_{4} m_{1 y}^{3}+a_{5} m_{1 x}^{2} m_{1 z}+a_{6} m_{1 y}^{2} m_{1 z} \\
& +a_{7} m_{1 x} m_{1 y} m_{1 z}+a_{8} m_{1 x} m_{1 z}^{2}+a_{9} m_{1 y} m_{1 z}^{2}+a_{10} m_{1 z}^{3}=0
\end{aligned}
$$

where the coefficients $a_{i}, i=1,2, \ldots, 10$, are functions of known parameters.

Similarly, for positions 1,2 , and 4 , we obtain the following equation by solving Eqs. (1), (2), and (10): 


$$
\begin{aligned}
\operatorname{det}\left(\mathbf{K}_{m}\right)_{124}= & \hat{\mathbf{M}}_{1} \cdot\left\{\left[\hat{\mathbf{s}}_{12} \times \hat{\mathbf{M}}_{1}+\tan \frac{\theta_{12}}{2}\left(\hat{\mathbf{s}}_{12} \cdot \hat{\mathbf{M}}_{1}\right) \hat{\mathbf{s}}_{12}\right]\right. \\
& \left.\times\left[\hat{\mathbf{s}}_{14} \times \hat{\mathbf{M}}_{1}+\tan \frac{\theta_{14}}{2}\left(\hat{\mathbf{s}}_{14} \cdot \hat{\mathbf{M}}_{1}\right) \hat{\mathbf{s}}_{14}\right]\right\} \\
= & 0
\end{aligned}
$$

Expanding the above equation gives

$$
\begin{aligned}
b_{1} m_{1 x} m_{1 y}^{2} & +b_{2} m_{1 x}^{2} m_{1 y}+b_{3} m_{1 x}^{3}+b_{4} m_{1 y}^{3}+b_{5} m_{1 x}^{2} m_{1 z}+b_{6} m_{1 y}^{2} m_{1 z} \\
& +b_{7} m_{1 x} m_{1 y} m_{1 z}+b_{8} m_{1 x} m_{1 z}^{2}+b_{9} m_{1 y} m_{1 z}^{2}+b_{10} m_{1 z}^{3}=0
\end{aligned}
$$

where the coefficients $b_{i}, i=1,2, \ldots, 10$, are functions of known parameters.

Next, we employ the dialytic elimination method [7] to seek a closed-form solution of $\hat{\mathbf{M}}_{1}\left(m_{1 x}, m_{1 y}, m_{1 z}\right)$. For the purpose of employing the dialytic elimination method, we denote Eqs. (17) and (19) with $f_{1}$ and $f_{2}$. In addition, to simplify the notations for the dialytic elimination process, let $m_{1 x}=x, m_{1 y}=y, m_{1 z}=z$. We have

$$
\begin{array}{r}
f_{1}: a_{1} x y^{2}+a_{2} x^{2} y+a_{3} x^{3}+a_{4} y^{3}+a_{5} x^{2} z+a_{6} y^{2} z \\
+a_{7} x y z+a_{8} x z^{2}+a_{9} y z^{2}+a_{10} z^{3}=0 \\
f_{2}: b_{1} x y^{2}+b_{2} x^{2} y+b_{3} x^{3}+b_{4} y^{3}+b_{5} x^{2} z+b_{6} y^{2} z \\
+b_{7} x y z+b_{8} x z^{2}+b_{9} y z^{2}+b_{10} z^{3}=0
\end{array}
$$

Note that $f_{1}$ and $f_{2}$ are homogeneous equations. Letting $y=p x$ and $z=q x$ and substituting them into $f_{1}$ and $f_{2}$ gives:

$$
\begin{array}{r}
f_{1}: a_{1} p^{2}+a_{2} p+a_{3}+a_{4} p^{3}+a_{5} q+a_{6} p^{2} q \\
+a_{7} p q+a_{8} q^{2}+a_{9} p q^{2}+a_{10} q^{3}=0 \\
f_{2}: b_{1} p^{2}+b_{2} p+b_{3}+b_{4} p^{3}+b_{5} q+b_{6} p^{2} q \\
+b_{7} p q+b_{8} q^{2}+b_{9} p q^{2}+b_{10} q^{3}=0
\end{array}
$$

Suppressing the variable $p$ into the coefficients gives:

$$
\begin{aligned}
& f_{1}: \mathrm{A}_{0} q^{3}+\mathrm{A}_{1} q^{2}+\mathrm{A}_{2} q+\mathrm{A}_{3}=0 \\
& f_{2}: \mathrm{B}_{0} q^{3}+\mathrm{B}_{1} q^{2}+\mathrm{B}_{2} q+\mathrm{B}_{3}=0
\end{aligned}
$$

Manipulating $f_{1}$ and $f_{2}$ in the following manners yields three equations:

$$
\begin{gathered}
f_{1} \times \mathrm{B}_{0}-f_{2} \times \mathrm{A}_{0}:\left(\mathrm{A}_{1} \mathrm{~B}_{0}-\mathrm{B}_{1} \mathrm{~A}_{0}\right) q^{2}+\left(\mathrm{A}_{2} \mathrm{~B}_{0}-\mathrm{B}_{2} \mathrm{~A}_{0}\right) q+\left(\mathrm{A}_{3} \mathrm{~B}_{0}-\mathrm{B}_{3} \mathrm{~A}_{0}\right)=0 \\
f_{1} \times \mathrm{B}_{3}-f_{2} \times \mathrm{A}_{3}:\left(\mathrm{A}_{0} \mathrm{~B}_{3}-\mathrm{B}_{0} \mathrm{~A}_{3}\right) q^{2}+\left(\mathrm{A}_{1} \mathrm{~B}_{3}-\mathrm{B}_{1} \mathrm{~A}_{3}\right) q+\left(\mathrm{A}_{2} \mathrm{~B}_{3}-\mathrm{B}_{2} \mathrm{~A}_{3}\right)=0 \\
f_{1} \times\left(\mathrm{B}_{0} \cdot q+\mathrm{B}_{1}\right)-f_{2} \times\left(\mathrm{A}_{0} \cdot q+\mathrm{A}_{1}\right):\left(\mathrm{A}_{2} \mathrm{~B}_{0}-\mathrm{A}_{0} \mathrm{~B}_{2}\right) q^{2} \\
+\left(\mathrm{A}_{2} \mathrm{~B}_{1}-\mathrm{A}_{1} \mathrm{~B}_{2}+\mathrm{A}_{3} \mathrm{~B}_{0}-\mathrm{A}_{0} \mathrm{~B}_{3}\right) q+\left(\mathrm{A}_{3} \mathrm{~B}_{1}-\mathrm{A}_{1} \mathrm{~B}_{3}\right)=0
\end{gathered}
$$


Rearranging Eqs. (26)-(28) in a matrix form gives:

$$
\left[\begin{array}{ccc}
\left(\mathrm{A}_{1} \mathrm{~B}_{0}-\mathrm{B}_{1} \mathrm{~A}_{0}\right) & \left(\mathrm{A}_{2} \mathrm{~B}_{0}-\mathrm{B}_{2} \mathrm{~A}_{0}\right) & \left(\mathrm{A}_{3} \mathrm{~B}_{0}-\mathrm{B}_{3} \mathrm{~A}_{0}\right) \\
\left(\mathrm{A}_{0} \mathrm{~B}_{3}-\mathrm{B}_{0} \mathrm{~A}_{3}\right) & \left(\mathrm{A}_{1} \mathrm{~B}_{3}-\mathrm{B}_{1} \mathrm{~A}_{3}\right) & \left(\mathrm{A}_{2} \mathrm{~B}_{3}-\mathrm{B}_{2} \mathrm{~A}_{3}\right) \\
\left(\mathrm{A}_{2} \mathrm{~B}_{0}-\mathrm{A}_{0} \mathrm{~B}_{2}\right) & \left(\mathrm{A}_{2} \mathrm{~B}_{1}-\mathrm{A}_{1} \mathrm{~B}_{2}+\mathrm{A}_{3} \mathrm{~B}_{0}-\mathrm{A}_{0} \mathrm{~B}_{3}\right) & \left(\mathrm{A}_{3} \mathrm{~B}_{1}-\mathrm{A}_{1} \mathrm{~B}_{3}\right)
\end{array}\right]\left[\begin{array}{c}
q^{2} \\
q \\
1
\end{array}\right]=0
$$

Again, to have non-trivial solutions of $q$, the determinant of the coefficient matrix must be zero, which gives a ninth degree polynomial equation in $p$. Upon substituting the solution of $p$ into Eq. (29), we obtain a corresponding $q$. Substituting the values of $p$ and $q$ into Eq. (5) gives:

$$
x=m_{1 x}=\sqrt{1 /\left(1+p^{2}+q^{2}\right)}
$$

which leads to the direction vector $\hat{\mathbf{M}}_{1}=\left(m_{1 x}, m_{1 y}, m_{1 z}\right)=(x, y, z)=(x, p x, q x)$.

Among the nine sets of solution of $p$ and $q$, there are two complex-number solutions satisfying the following constraint:

$$
p^{2}+q^{2}=-1
$$

which leads to two infinite solutions of $\hat{\mathbf{M}}_{1}\left(m_{1 x}, m_{1 y}, m_{1 z}\right)$. One of the remaining seven sets of solution is $\hat{\mathbf{s}}_{12}\left(s_{12 x}, s_{12 y}, s_{12 z}\right)$, which cannot be used for the moving joint. Therefore, we have a total of six finite solutions of $\hat{\mathbf{M}}_{1}\left(m_{1 x}, m_{1 y}, m_{1 z}\right)$. Substituting each solution of $\hat{\mathbf{M}}_{1}\left(m_{1 x}, m_{1 y}, m_{1 z}\right)$ into Eqs. (1), (2), and (6) gives a corresponding $\hat{\mathbf{F}}\left(f_{x}, f_{y}, f_{z}\right)$.

\section{Solutions of Position Vectors and Numerical Example}

Once the solutions of direction vectors are obtained, we can substitute them into Eqs. (3), (4), (7), (8), (11), and (12) to solve for the position vectors $\mathbf{Q}_{1}\left(q_{1 x}, q_{1 y}, q_{1 z}\right)$ and $\mathbf{G}\left(g_{x}, g_{y}, g_{z}\right)$. First, we rearrange Eqs. (3), (4), (8), and (11) in to a matrix form as follows:

$$
\left[\mathbf{K}_{q}\right]\left[\begin{array}{c}
g_{x} \\
g_{y} \\
g_{z} \\
1
\end{array}\right]=\left[\begin{array}{c}
0 \\
0 \\
0 \\
0
\end{array}\right]
$$

where $\mathbf{K}_{q}$ is of the following form

$$
\mathbf{K}_{q}=\left[\begin{array}{cccc}
f_{x} & f_{y} & f_{z} & 0 \\
k_{q 21} & k_{q 22} & k_{q 23} & k_{q 24} \\
k_{q 31} & k_{q 32} & k_{q 33} & k_{q 34} \\
k_{q 41} & k_{q 42} & k_{q 43} & k_{q 44}
\end{array}\right]
$$


In order for Eq. (32) to have non-trivial solutions, the determinant of $\mathbf{K}_{q}$ must be zero, which gives the following linear equation in $\left(q_{1 x}, q_{1 y}, q_{1 z}\right)$ :

$$
k_{1} q_{1 x}+k_{2} q_{1 y}+k_{3} q_{1 z}+k_{4}=0
$$

where the coefficients $k_{i}, i=1,2,3,4$, contain the specified parameters and the direction vectors previously solved. Similarly, we obtain another linear equation by using Eqs. (3), (4), (8), and (12):

$$
k_{5} q_{1 x}+k_{6} q_{1 y}+k_{7} q_{1 z}+k_{8}=0
$$

where the coefficients $k_{i}, i=5,6,7,8$, contain the specified parameters and the direction vectors previously solved. Now we have three linear equations, Eqs. (7), (34), and (35), allowing us to obtain a unique solution of $\left(q_{1 x}, q_{1 y}, q_{1 z}\right)$. Finally, we can use Eqs. (32) to obtain the corresponding solution of $\left(g_{x}, g_{y}, g_{z}\right)$.

In what follows, we provide a numerical example to verify the solution procedure discussed above. Table 1 gives three displacement screws denoting four positions of a body. Following the discussed solution procedure, we obtain six real solutions, as listed in Table 2. Note that in this case, we have six real solutions, while other specifications may yield only four, two, or zero real solutions.

Table 1 Displacement screws for the four specified positions

\begin{tabular}{ccccc}
\hline Screw & Direction vector & Position vector of a point & Translation & Rotation \\
\hline 12 & $(0.103,0.737,-0.668)$ & $(0.941,0.521,-0.419)$ & 1.384 & $183.616^{\circ}$ \\
13 & $(-0.208,-0.838,-0.505)$ & $(-0.610,0.837,0.123)$ & 1.899 & $178.431^{\circ}$ \\
14 & $(-0.832,0.261,0.490)$ & $(0.733,0.689,-0.164)$ & -1.117 & $234.494^{\circ}$ \\
\hline
\end{tabular}

\section{Conclusion}

This paper presents the solution of the spatial slider for guiding a rigid body to pass through four positions. By using screw triangle geometry, we obtained 12 equations to solve for 12 unknowns that determine a perpendicularly intersected cylindricalcylindrical dyad. In order to seek a closed-form solution, we decoupled the equations into two groups and solved the direction vectors using one of the groups. The position vectors of points on the cylindrical joint axes were obtained by solving the second group of design equations.

By using the dialytic elimination method, we obtained a ninth degree polynomial equation that led to nine possible solutions. Among the nine sets of solutions, two of them are infinite, and one is the displacement screw from the first position to the second position. Therefore, we have at most six real solutions that can be used to 
Table 2 Solution to the synthesis equations

\begin{tabular}{ccc}
\hline Solution\# & $\hat{\mathbf{F}}\left(f_{x}, f_{y}, f_{z}\right)$ & $\mathbf{G}\left(g_{x}, g_{y}, g_{z}\right)$ \\
\hline 1 & $(-0.6917,0.5429,0.4762)$ & $(0.2110,0.1513,0.1341)$ \\
2 & $(0.3975,0.5431,0.7396)$ & $(1.9873,0.4768,-1.4183)$ \\
3 & $(-0.3927,0.7106,0.5838)$ & $(-0.8295,0.7632,-1.4870)$ \\
4 & $(-0.1186,-0.5320,0.8384)$ & $(-6.4081,-1.8656,-2.0903)$ \\
5 & $(-0.9773,0.1780,0.1149)$ & $(0.2275,0.9293,0.4961)$ \\
6 & $(-0.0933,-0.6980,0.7100)$ & $(7.5762,-1.0020,0.0105)$ \\
\hline & $\hat{\mathbf{M}}_{1}\left(m_{1 x}, m_{1 y}, m_{1 z}\right)$ & $\mathbf{Q}_{1}\left(q_{1 x}, q_{1 y}, q_{1 z}\right)$ \\
\hline 1 & $(-0.1323,0.5529,-0.8227)$ & $(-1.6848,1.6638,1.3892)$ \\
2 & $(-0.8434,-0.1013,0.5277)$ & $(-0.1331,0.1786,-0.1784)$ \\
3 & $(-0.1029,-0.7372,0.6678)$ & $(-1.8024,0.9896,0.8147)$ \\
4 & $(0.9900,0.0011,0.1407)$ & $(-0.9567,-6.8947,6.7816)$ \\
5 & $(0.2009,0.9508,0.2358)$ & $(-1.3157,0.1772,0.4061)$ \\
6 & $(0.9863,-0.1622,-0.0298)$ & $(0.6223,4.5468,-4.1465)$ \\
\hline
\end{tabular}

design spatial sliders for four-position problems. Notice that the solution procedure and result given in this paper are comparable to those in the synthesis of a planar slider.

\section{References}

1. Bottema, O. and Roth, B.: Theoretical Kinematics. Dover Publications, Inc., New York (1990)

2. Burmester, L.: Geraführung durch das Kurbelgetriebe. Civilingenieru, 22, 597-606 (1876)

3. Roth, B.: On the Screw Axes and Other Special Lines Associated with Spatial Displacements of a Rigid Body. Journal of Engineering for Industry, Trans. ASME, B, 102-110 (1967)

4. Tsai, L. W., and Roth, B.: Design of Dyads with Helical, Cylindrical, Spherical, Revolute and Prismatic Joints. Mechanism and Machine Theory, 7, 85-102 (1972)

5. Larochelle, P. M.: Circuit and Branch Rectification of the Spatial 4C Mechanism. Proc. ASME DETC Mechanism Conf., Baltimore, Maryland, USA, September 10-13 (2000)

6. Murray, A. P., and McCarthy, J. M.: Five Position Synthesis of Spatial CC Dyad. Proc. ASME DETC Mechanism Conf., Minneapolis, Minnesota, USA, September 11-14 (1994)

7. Tsai, L. W.: Robot Analysis: The Mechanics of Serial and Parallel Manipulator. John Wiley \& Sons, New York (1999)

8. Wu, C. H.: Spatial Generalization of the Synthesis of Planar Slider Linkages. M.S. Thesis, Department of Mechanical Engineering, National Cheng Kung University, Taiwan (2011) 